\title{
Natural variables for density functionals
}

\author{
Yan Alexander Wang \\ Department of Chemistry, University of North Carolina, Chapel Hill, North Carolina 27599
}

(Received 25 November 1996)

\begin{abstract}
Various energy functionals in density-functional theory must possess the form of $\left\langle\beta^{3+\mu} f(\zeta\right.$, $\left.\left.\eta, \xi_{1}, \xi_{2}, \ldots, \xi_{n}\right)\right\rangle$, if one assumes that they can be expressed as $\left\langle g\left(\lambda, \mathbf{r}, \rho(\mathbf{r}), \rho^{(1)}, \rho^{(2)}, \ldots, \rho^{(n)}\right)\right\rangle$. Here $\rho(\mathbf{r})$ and $\rho^{(m)}$ are the electron density and its $m$ th-order gradient; $f$ and $g$ are proper analytic functions of their variables; $\mu$ is 2 when the functionals are $T_{s}[\rho], T_{c}^{\lambda}[\rho]$, and $T^{\lambda}[\rho]$ (otherwise it equals 1 ); $\lambda$ is the electron-electron interaction coupling constant in the adiabatic connection formulation; and $\left\{\beta, \zeta, \eta, \xi_{1}, \xi_{2}, \ldots, \xi_{n}\right\}$ is a set of independent variables (called natural variables) in place of $\left\{\lambda, \mathbf{r}, \rho, \rho^{(1)}, \rho^{(2)}, \ldots, \rho^{(n)}\right\}$. These variables are defined as $\left\{\beta(\mathbf{r})=\rho^{1 / 3}(\mathbf{r}), \zeta=\beta / \lambda, \eta=\mathbf{r} \beta, \xi_{m}=\rho^{(m)} / \beta^{3+m} \mid m=1,2, \ldots, n\right\}$. Generalizations to more complex functional forms are also discussed. Some exact relationships are derived that should be useful for developing density functionals of the weighted-density approximation type. [S1050-2947(97)01806-4]
\end{abstract}

PACS number(s): 31.15.Ew

The immense success of the modern density-functional theory (DFT) $[1,2]$ originated from two papers $[3,4]$ by Hohenberg, Kohn, and Sham more than three decades ago, in which the electron density $\rho(\mathbf{r})$ was legitimatized as the basic variable of ground-state quantum chemistry. However, the existence of the Hohenberg-Kohn (HK) theorems [3] does not help much in the construction of the exact total-energy functional (hence its components) from $\rho(\mathbf{r})$. From the later 1970s on, the adiabatic connection formulation [5-7] and later the constrained-search formulation $[8,9]$ have nourished much of the advancement in DFT.

Via the constrained-search formulation [8,9], the HK universal functional $[3] F_{\lambda}[\rho]$, defined within an extended domain

$$
F_{\lambda}[\rho]=\left\langle\Psi^{\lambda}\left|\hat{T}+\lambda \hat{V}_{e e}\right| \Psi^{\lambda}\right\rangle,
$$

always has a minimum [10] for an antisymmetric $N$-electron wave function $\Psi^{\lambda}$, with a specific electronelectron interaction coupling constant $\lambda$ (which must be set equal to 1 for realistic full Coulomb systems). Here $\Psi^{\lambda}$ generates an $N$ - and $v$-representable $\rho(\mathbf{r})$ and is an eigenstate of the coupled Hamiltonian [11]

$$
\hat{H}_{\lambda}=\hat{T}+\lambda \hat{V}_{e e}+\hat{V}_{\mathrm{ext}}^{\lambda},
$$

where $\hat{T}, \hat{V}_{e e}$, and $\hat{V}_{\mathrm{ext}}^{\lambda}$ are the kinetic energy, the electronelectron repulsion, and the external potential operators, respectively. In the spirit of the Kohn-Sham theory [4], $F_{\lambda}[\rho]$ is partitioned into three main pieces

$$
F_{\lambda}[\rho]=T_{s}[\rho]+\lambda J[\rho]+\lambda E_{\mathrm{xc}}^{\lambda}[\rho],
$$

where $T_{s}[\rho]$ is the noninteracting $(\lambda=0)$ kinetic-energy functional, $J[\rho]$ is the classical electron-electron Coulomb repulsion functional, and $E_{\mathrm{xc}}^{\lambda}[\rho]$ is the exact exchangecorrelation functional. $E_{\mathrm{xc}}^{\lambda}[\rho]$ in turn can be decomposed into two components [12]

$$
E_{\mathrm{xc}}^{\lambda}[\rho]=E_{x}[\rho]+E_{c}^{\lambda}[\rho],
$$

namely, the $\lambda$-independent exchange functional $E_{x}[\rho]$,

$$
E_{x}[\rho]=\left\langle\Psi^{\lambda=0}\left|\hat{V}_{e e}\right| \Psi^{\lambda=0}\right\rangle-J[\rho]=V_{e e}^{0}[\rho]-J[\rho],
$$

and the $\lambda$-dependent correlation functional $E_{c}^{\lambda}[\rho]$,

$$
E_{c}^{\lambda}[\rho]=(1 / \lambda) T_{c}^{\lambda}[\rho]+V_{c}^{\lambda}[\rho]
$$

where

$$
T_{c}^{\lambda}[\rho]=\left\langle\Psi^{\lambda}|\hat{T}| \Psi^{\lambda}\right\rangle-\left\langle\Psi^{\lambda=0}|\hat{T}| \Psi^{\lambda=0}\right\rangle=T^{\lambda}[\rho]-T_{s}[\rho],
$$

and

$$
\begin{aligned}
V_{c}^{\lambda}[\rho] & =\left\langle\Psi^{\lambda}\left|\hat{V}_{e e}\right| \Psi^{\lambda}\right\rangle-\left\langle\Psi^{\lambda=0}\left|\hat{V}_{e e}\right| \Psi^{\lambda=0}\right\rangle \\
& =V_{e e}^{\lambda}[\rho]-V_{e e}^{0}[\rho] .
\end{aligned}
$$

The following exact relations have been established for the $J[p], E_{x}[\rho], E_{c}^{\lambda}[\rho], E_{\mathrm{xc}}^{\lambda}[\rho], T_{s}[\rho], T_{c}^{\lambda}[\rho], T^{\lambda}[\rho]$, $V_{e e}^{0}[\rho], V_{c}^{\lambda}[\rho]$, and $V_{e e}^{\lambda}[\rho]$ functionals [12-15]:

$$
\begin{gathered}
\mu G\left[\rho_{;} \lambda\right]=\lambda d G\left[\rho_{;} \lambda\right] / d \lambda+\hat{S}_{c} G[\rho ; \lambda], \\
T_{c}^{\lambda}[\rho]=d E_{c}^{\lambda}[\rho] / d(1 / \lambda)=d E_{\mathrm{xc}}^{\lambda}[\rho] / d(1 / \lambda),
\end{gathered}
$$

and

$$
\frac{d V_{e e}^{\lambda}[\rho]}{d \lambda}=\frac{d V_{c}^{\lambda}[\rho]}{d \lambda}=-\frac{1}{\lambda} \frac{d T_{c}^{\lambda}[\rho]}{d \lambda}=-\frac{1}{\lambda} \frac{d T^{\lambda}[\rho]}{d \lambda} .
$$

Here

$$
\hat{S}_{c}=-\int d \tau \rho(\mathbf{r})(\mathbf{r} \cdot \nabla)[\delta / \delta \rho(\mathbf{r})]
$$

is the functional coordinate-scaling operator [15] and $G\left[\rho_{;} \lambda\right]$ is a dummy density functional in place of any of those just mentioned above. In Eq. (9), $\mu$ is 2 for $T_{s}[\rho]$, $T_{c}^{\lambda}[\rho]$, and $T^{\lambda}[\rho]$ (otherwise, it equals 1 ), and the first term of the right-hand side (RHS) involving $\lambda$ will vanish automatically if $G\left[\rho_{;} \lambda\right]$ is $\lambda$ independent, i.e.,

$$
G[\rho, \lambda]=G[\rho] .
$$

For later use, one defines the $m$ th-order gradient of $\rho(\mathbf{r})$ as

$$
\rho^{(m)} \equiv \nabla^{m} \rho(\mathbf{r}) \equiv \sum_{k} \rho_{k}^{(m)}
$$


and a compact notation for an arbitrary analytic function $g\left(\rho^{(m)}\right)$,

$$
\rho^{(m)}\left(\partial g / \partial \rho^{(m)}\right) \equiv \sum_{k} \rho_{k}^{(m)}\left(\partial g / \partial \rho_{k}^{(m)}\right),
$$

where $\rho_{k}^{(m)}$ denotes one component of $\rho^{(m)}$,

$$
\rho_{k}^{(m)} \equiv \frac{\partial^{m} \rho(\mathbf{r})}{\left(\partial x_{k}\right)^{m}} \equiv \frac{\partial^{m} \rho(\mathbf{r})}{\partial x_{k 1} \partial x_{k 2} \cdots \partial x_{k m}} .
$$

Here $\left\{x_{k 1}, x_{k 2}, \ldots, x_{k m}\right\}$ is a specific (the $k$ th) combination of the three coordinate indices $\{x, y, z\}$ and the unit vectors along axis directions are stripped from Eq. (14) for simplicity. More generally, for an entity

$$
\theta \equiv \sum_{k} \theta_{k}
$$

and its arbitrary analytic function $g(\theta)$, there is a compact notation

$$
\theta(\partial g / \partial \theta) \equiv \sum_{k} \theta_{k}\left(\partial g / \partial \theta_{k}\right) .
$$

Unless otherwise noticed hereafter, the Einstein summation convention will be applied over repeated indices.

Lemma. Assume a general density functional of the form

$$
G\left[\rho_{;} \lambda\right]=\left\langle g\left(\lambda, \mathbf{r}, \rho, \rho^{(1)}, \rho^{(2)}, \ldots, \rho^{(n)}\right)\right\rangle,
$$

where $g$ is an analytic function of its variables, and $\rho$ and its gradients are strongly vanishing asymptotically. Then the following identities hold:

$$
\begin{aligned}
3 G\left[\rho_{;} \lambda\right]+\left\langle(\mathbf{r} \cdot \boldsymbol{\nabla}) \rho \mid \frac{\delta G}{\delta \rho}\right\rangle & =m\left\langle\rho^{(m)} \mid \frac{\partial g}{\partial \rho^{(m)}}\right\rangle-\left\langle\mathbf{r} \mid \frac{\partial g}{\partial \mathbf{r}}\right\rangle, \\
3 G\left[\rho_{;} \lambda\right]+\hat{S}_{c} G\left[\rho_{;} \lambda\right]= & 3\left\langle\rho \mid \frac{\partial g}{\partial \rho}\right\rangle+(3+m)\left\langle\rho^{(m)} \mid \frac{\partial g}{\partial \rho^{(m)}}\right\rangle \\
& -\left\langle\mathbf{r} \mid \frac{\partial g}{\partial \mathbf{r}}\right\rangle,
\end{aligned}
$$

and

$$
\begin{aligned}
(3+\mu) G[\rho ; \lambda]-3\left\langle\rho \mid \frac{\partial g}{\partial \boldsymbol{\rho}}\right\rangle= & (3+m)\left\langle\boldsymbol{\rho}^{(m)} \mid \frac{\partial g}{\partial \boldsymbol{\rho}^{(m)}}\right\rangle \\
& -\left\langle\mathbf{r} \mid \frac{\partial g}{\partial \mathbf{r}}\right\rangle+\lambda \frac{d G}{d \lambda} .
\end{aligned}
$$

Proof. For a density functional defined as Eq. (19), its functional derivative is [1]

$$
\frac{\delta G}{\delta \rho}=\frac{\partial g}{\partial \rho}+(-1)^{m} \frac{\partial^{m}}{\left(\partial x_{k}\right)^{m}} \frac{\partial g}{\partial \rho_{k}^{(m)}} .
$$

With successive integration by parts, one can readily show that

$$
\left\langle\rho \mid \frac{\delta G}{\delta \rho}\right\rangle=\left\langle\rho \mid \frac{\partial g}{\partial \rho}\right\rangle+\left\langle\rho^{(m)} \mid \frac{\partial g}{\partial \rho^{(m)}}\right\rangle .
$$

Following a previous work [16], $G[\rho ; \lambda]$ can be transformed by an integration by parts to

$$
\begin{aligned}
-3 G\left[\rho_{;} \lambda\right]= & \left\langle\left(\mathbf{r} \cdot \nabla_{T}\right) g\right\rangle=\left\langle\mathbf{r} \frac{\partial g}{\partial \mathbf{r}}\right\rangle+\left\langle(\mathbf{r} \cdot \nabla) \rho \mid \frac{\partial g}{\partial \rho}\right\rangle \\
& +\left\langle x_{j} \frac{\partial g}{\partial \rho_{k}^{(m)}} \frac{\partial \rho_{k}^{(m)}}{\partial x_{j}}\right\rangle,
\end{aligned}
$$

where $\boldsymbol{\nabla}_{T}$ denotes the total derivative with respect to $\mathbf{r}$. Combination of Eqs. (19) and (23)-(25) and more integrations by parts yield the identity of Eq. (20). Here Eqs. (20) and (25) are generalizations (to arbitrary order) of the results (only first order) derived earlier [16]. A simple integration by parts shows that, in general,

$$
\hat{S}_{c} G\left[\rho_{;} \lambda\right]=3\left\langle\rho \mid \frac{\partial G}{\partial \rho}\right\rangle+\left\langle(\mathbf{r} \cdot \nabla) \rho \mid \frac{\delta G}{\delta \rho}\right\rangle .
$$

Combination of Eqs. (20), (24), and (26) yields the identity of Eq. (21), and elimination of the $\hat{S}_{c}$ terms from Eqs. (9) and (21) delivers Eq. (22). Q.E.D.

From this lemma, there follows a simple theorem, which can aid in the invention of new density functionals and serve as a criterion for judging the correctness of approximate ones.

Theorem 1. The variables

$$
\left\{\beta(\mathbf{r})=\rho^{1 / 3}(\mathbf{r}), \quad \begin{array}{c}
\beta=\beta / \lambda, \quad \eta=\mathbf{r} \beta, \quad \xi_{m}=\rho^{(m)} / \beta^{3+m} \mid \\
m=1,2, \ldots, n\}
\end{array}\right.
$$

are natural variables of DFT which act as independent variables in place of $\left\{\lambda, \mathbf{r}, \rho(\mathbf{r}), \rho^{(1)}, \rho^{(2)}, \ldots, \rho^{(n)}\right\}$ in Eq. (19), such that for any density functional satisfying Eqs. (9) and (19) -(22) there exists

$$
g\left(\lambda, \mathbf{r}, \rho, \rho^{(1)}, \rho^{(2)}, \ldots, \rho^{(n)}\right) \equiv \beta^{3+\mu} f\left(\zeta, \eta, \xi_{1}, \xi_{2}, \ldots, \xi_{n}\right)
$$

and hence

$$
G\left[\rho_{;} \lambda\right] \equiv\left\langle\beta^{3+\mu} f\left(\zeta, \eta, \xi_{1}, \xi_{2}, \ldots, \xi_{n}\right)\right\rangle,
$$

where $f$ is an analytic function of its variables.

Proof. After transforming the variables according to Eq. (27), one has

$$
\begin{gathered}
\lambda \frac{d G}{d \lambda}=-\left\langle\zeta \mid \frac{\partial g}{\partial \zeta}\right\rangle, \\
\left\langle\mathbf{r} \mid \frac{\partial g}{\partial \mathbf{r}}\right\rangle=\left\langle\eta \mid \frac{\partial g}{\partial \eta}\right\rangle, \\
\left\langle\boldsymbol{\rho}^{(m)} \mid \frac{\partial g}{\partial \rho^{(m)}}\right\rangle=\left\langle\xi_{m} \mid \frac{\partial g}{\partial \xi_{m}}\right\rangle .
\end{gathered}
$$

Further, with Eqs. (28) and (29), one has

$$
\begin{aligned}
3\left\langle\rho \mid \frac{\partial g}{\partial \rho}\right\rangle= & 3\left\langle\rho \mid \frac{\partial g}{\partial \beta} \frac{\partial \beta}{\partial \rho}\right\rangle+3\left\langle\rho \mid \frac{\partial g}{\partial \zeta} \frac{\partial \zeta}{\partial \rho}\right\rangle \\
& +3\left\langle\rho \mid \frac{\partial g}{\partial \eta} \frac{\partial \eta}{\partial \rho}\right\rangle+3\left\langle\rho \mid \frac{\partial g}{\partial \xi_{m}} \frac{\partial \xi_{m}}{\partial \rho}\right\rangle \\
= & (3+\mu) G\left[\rho_{;} \lambda\right]+\left\langle\zeta \mid \frac{\partial g}{\partial \zeta}\right\rangle \\
& +\left\langle\eta \mid \frac{\partial g}{\partial \eta}\right\rangle-(3+m)\left\langle\xi_{m} \mid \frac{\partial g}{\partial \xi_{m}}\right\rangle .
\end{aligned}
$$

Hence, for Eq. (22), one obtains that 


$$
(\mathrm{LHS}) \equiv(\mathrm{RHS})=-\left\langle\zeta \mid \frac{\partial g}{\partial \zeta}\right\rangle-\left\langle\eta \mid \frac{\partial g}{\partial \eta}\right\rangle+(3+m)\left\langle\xi_{m} \mid \frac{\partial g}{\partial \xi_{m}}\right\rangle
$$

Therefore, Eq. (29) is a general solution of the exact relations (9) and (20)-(22). Further, one can easily prove that any departure from Eqs. (27)-(29) will destroy the identical equality in Eq. (34); hence the variables defined by Eq. (27) are natural variables of DFT. Q.E.D.

Clearly, any exact density functional can always be brought into the form of Eqs. (28) and (29). Therefore, the use of the natural variables in the construction of any approximate density functionals will automatically ensure them to satisfy Eqs. (9) and (20)-(22). Moreover, any general $G\left[\rho_{;} \lambda\right]$ can be obtained from $G\left[\rho_{;} \lambda=1\right]$ with the aid of Theorem 1. For instance, the classical Wigner correlation functional [17] can be written as

$$
E_{c}^{\lambda=1}[\rho]_{\text {Wigner }}=\left\langle a \beta^{4} /(1+b \beta)\right\rangle,
$$

where $\{a, b\}$ are fitting parameters. With Theorem 1, one immediately gets $[18,19]$

$$
E_{c}^{\lambda}[\rho]_{\text {Wigner }}=\left\langle a \beta^{4} /(1+b \zeta)\right\rangle,
$$

which can then be used to generate $T_{c}^{\lambda}[\rho]$ and $V_{c}^{\lambda}[\rho]$ via Eqs. (6) and (10).

Generalizations of Theorem 1 to more complex functional forms result in the following theorem and corollary to it.

Theorem 2. Assume a general density functional of the form

$$
G[\rho ; \lambda]=\left\langle\left\langle g\left(\lambda, \omega_{1}, \omega_{2}\right)\right\rangle\right\rangle,
$$

where the variable set $\omega_{i}$ is

$$
\omega_{i} \equiv\left\{\mathbf{r}_{i}, \rho_{i}, \rho_{i}^{(1)}, \rho_{i}^{(2)}, \ldots, \rho_{i}^{(n)}\right\} .
$$

It is understood here that the subscripts are electron indices, the double angular brackets are integrations over the coordinates of two electrons, $g$ is a symmetric analytic function of its variables, $\rho_{i}$ and its gradients are strongly vanishing asymptotically. Then, the following identities hold:

$$
\begin{aligned}
& \frac{\delta G}{\delta \rho(\mathbf{r})}=\left\langle\left\langle\left(\frac{\partial g}{\partial \rho_{i}}+(-1)^{m} \frac{\partial^{m}}{\left(\partial x_{i k}\right)^{m}} \frac{\partial g}{\partial \boldsymbol{\rho}_{i k}^{(m)}}\right) \delta\left(\mathbf{r}_{i}-\mathbf{r}\right)\right\rangle\right\rangle, \\
& \left\langle\rho \mid \frac{\delta G}{\delta \rho}\right\rangle=\left\langle\left\langle\rho_{i} \mid \frac{\partial g}{\partial \rho_{i}}\right\rangle\right)+\left\langle\left\langle\rho_{i}^{(m)} \mid \frac{\partial g}{\partial \rho_{i}^{(m)}}\right\rangle\right\rangle, \\
& -6 G\left[\rho_{;} \lambda\right]=\left\langle\left\langle\mathbf{r}_{i} \mid \frac{\partial g}{\partial \mathbf{r}_{i}}\right\rangle\right)+\left\langle\left\langle\left(\mathbf{r}_{i} \cdot \nabla_{i}\right) \rho_{i} \mid \frac{\partial g}{\partial \rho_{i}}\right\rangle\right\rangle \\
& +\left\langle\left\langle x_{i j} \frac{\partial g}{\partial \rho_{i k}^{(m)}} \frac{\partial \rho_{i k}^{(m)}}{\partial x_{i j}}\right\rangle\right\rangle \text {, } \\
& 6 G\left[\rho_{;} \lambda\right]+\left\langle(\mathbf{r} \cdot \boldsymbol{\nabla}) \rho \mid \frac{\delta G}{\delta \rho}\right\rangle=m\left\langle\left\langle\rho_{i}^{(m)} \mid \frac{\partial g}{\partial \rho_{i}^{(m)}}\right\rangle\right\rangle \\
& -\left\langle\left\langle\mathbf{r}_{i} \mid \frac{\partial g}{\partial \mathbf{r}_{i}}\right\rangle\right),
\end{aligned}
$$

$6 G[\rho ; \lambda]+\hat{S}_{c} G\left[\rho_{;} \lambda\right]$

$$
=3\left\langle\left\langle\rho_{i} \mid \frac{\partial g}{\partial \rho_{i}}\right\rangle\right\rangle+(3+m)\left\langle\left\langle\rho_{i}^{(m)} \mid \frac{\partial g}{\partial \rho_{i}^{(m)}}\right\rangle\right)-\left\langle\left\langle\mathbf{r}_{i} \mid \frac{\partial g}{\partial \mathbf{r}_{i}}\right\rangle\right\rangle,
$$

and

$$
\begin{aligned}
\left.(6+\mu) G\left[\rho_{;} \lambda\right]-3\left\langle\left\langle\rho_{i}\right| \frac{\partial g}{\partial \rho_{i}}\right)\right\rangle= & (3+m)\left\langle\left\langle\rho_{i}^{(m)} \mid \frac{\partial g}{\partial \rho_{i}^{(m)}}\right\rangle\right) \\
& -\left\langle\left\langle\mathbf{r}_{i} \mid \frac{\partial g}{\partial \mathbf{r}_{i}}\right\rangle\right\rangle+\lambda \frac{d G}{d \lambda}
\end{aligned}
$$

This theorem is given here without detailed proof since it is similar to the arguments used earlier.

In the language of the pair correlation function and the exchange-correlation hole $[1,2]$, one has the following corollary.

Corollary. Assume that in Eq. (37),

$$
\begin{aligned}
g\left(\lambda, \omega_{1}, \omega_{2}\right) & =\left(\rho_{1} \rho_{2} / r_{12}\right) h\left(\lambda, \rho_{1}, \rho_{2}, r_{12}\right) \\
& =w\left(\rho_{1}, \rho_{2}, r_{12}\right) h\left(\lambda, \rho_{1}, \rho_{2}, r_{12}\right),
\end{aligned}
$$

where $r_{12}$ is the interelectronic distance and $h$ is a symmetric analytic function of its variables. Then $h$ has to satisfy the equation

$$
\begin{aligned}
& (\mu-1) G\left[\rho_{;} \lambda\right]-\left\langle\left\langle\beta_{i} \frac{\partial h}{\partial \beta_{i}} \mid w\right\rangle\right) \\
& =\left\langle\left\langle\lambda \frac{\partial h}{\partial \lambda} \mid w\right\rangle\right\rangle-\left\langle\left\langle r_{12} \frac{\partial h}{\partial r_{12}} \mid \psi\right\rangle\right\rangle .
\end{aligned}
$$

Further, $h$ can be chosen such that the integrands above on both sides are equal:

$$
(\mu-1) h-\beta_{i}\left(\partial h / \partial \beta_{i}\right)=\lambda(\partial h / \partial \lambda)-r_{12}\left(\partial h / \partial r_{12}\right) .
$$

Proof of this corollary is omitted here, since it is just an elementary application of Theorem 2. During the derivation of Eq. (46) from Eq. (44), one needs the two identities

$$
\mathbf{r}(\partial / \partial \mathbf{r}) \equiv \mathbf{r} \cdot \boldsymbol{\nabla}=r(\partial / \partial r)
$$

and

$$
r_{1}\left(\partial h / \partial r_{1}\right)+r_{2}\left(\partial h / \partial r_{2}\right)=r_{12}\left(\partial h / \partial r_{12}\right) \text {. }
$$

Due to the unknown relationship between $\beta_{1}$ and $\beta_{2}$, the effort to pin down the natural variables for Eqs. (37) and (45) is elusive. For example, among plausible candidates, $h$ can be written as

$$
h\left(\lambda, \rho_{1}, \rho_{2}, r_{12}\right)=\beta_{12}^{\mu-1} t\left(\beta_{12} / \lambda, r_{12} \beta_{12}\right),
$$

where $t$ is an analytic function and $\beta_{12}$ can be any symmetric analytic function of $\beta_{1}$ and $\beta_{2}$ [e.g., $\left(\beta_{1} \beta_{2}\right)^{1 / 2}$ or $\left(\beta_{1}^{\nu}\right.$ $\left.\left.+\beta_{2}^{\nu}\right)^{1 / \nu}\right]$ as long as it satisfies

$$
\beta_{1}\left(\partial \beta_{12} / \partial \beta_{1}\right)+\beta_{2}\left(\partial \beta_{12} / \partial \beta_{2}\right)=\beta_{12}
$$

in Eqs. (46) and (47). More studies ought to be carried out on the function forms of $g\left(\lambda, \omega_{1}, \omega_{2}\right)$ and $h\left(\lambda, \rho_{1}, \rho_{2}, r_{12}\right)$.

By the corollary, a requirement on $h\left(\lambda, \rho_{1}, \rho_{2}, r_{12}\right)$ is that $\rho_{1}$ and $\rho_{2}$ must be explicitly included along with $r_{12}$; otherwise Eq. (46) and, more strictly, Eq. (47) would not be true in general (e.g., for $E_{x}[\rho]$ ). This is at variance with the conventional weighted-density approximation (WDA) [20,21], in which one only assumes a nonsymmetric $h$ such that

$$
g\left(\lambda, \omega_{1}, \omega_{2}\right)=\left(\rho_{1} \rho_{2} / r_{12}\right) h\left(\lambda, \rho_{1}, r_{12}\right) .
$$


New generations of the WDA, which might be called the generalized WDA (GWDA), can be designed in accordance with Theorem 2 and its corollary. For instance, one possible GWDA scheme would employ simple symmetrization of Eq. (52):

$$
g\left(\lambda, \omega_{1}, \omega_{2}\right)=\frac{\rho_{1} \rho_{2}}{r_{12}} \frac{h\left(\lambda, \rho_{1}, r_{12}\right)+h\left(\lambda, \rho_{2}, r_{12}\right)}{2} .
$$

Table I lists some approximations to the exact expression in Eqs. (29) and (37), namely, the local-density approximation (LDA) [13,15,17-19,22-24], the reduced weighteddensity approximation (RWDA), the WDA [20,21], the GWDA, the gradient expansion approximation (GEA) [2531] and the generalized-gradient approximation (GGA) [19,31-40]. It is interesting to note that the RWDA and the GWDA are different from the classical WDA [20,21]. Numerical performance of the RWDA and the GWDA schemes should be tested. Moreover, Theorem 1 points out new directions in the effort to generate better approximate functionals by either including more higher-order gradient terms or combining the merits of the RWDA and the GGA/GEA schemes. One may further point out that in order to fix the correct or approximate functional forms, this work should be employed along with scaling properties and other exact relations [e.g., Eqs. (10) and (11)] of the DFT functionals [12-
TABLE I. Some approximations to the exact expressions of Eqs. (29) and (37). The interelectronic coupling constant $\lambda$ will be set to 1 for realistic full Coulomb systems. The definitions of the variables are shown in Eq. (27).

\begin{tabular}{lcc}
\hline \hline Approximation & Functional forms & References \\
\hline LDA & $\left\langle\beta^{3+\mu} f(\zeta)\right\rangle$ & {$[13,15,17-19,22-24]$} \\
GEA & $\left\langle\beta^{3+\mu} f\left(\zeta, \xi_{1}, \xi_{2}, \xi_{3}\right)\right\rangle$ & {$[25-31]$} \\
GGA & $\left\langle\beta^{3+\mu} f\left(\zeta, \xi_{1}\right)\right\rangle$ & {$[19,31-40]$} \\
RWDA & $\left\langle\beta^{3+\mu} f(\zeta, \eta)\right\rangle$ & this work \\
WDA & Eq. $(52)$ & {$[20,21]$} \\
GWDA & Eqs. (37), (45), & this work \\
& $(50)$, and $(53)$ & \\
\hline
\end{tabular}

$15,18,19,31,37$. Finally, the work presented here not only confirms but also generalizes the naive dimensional analysis [1].

This research was supported by a grant from the National Science Foundation to the University of North Carolina at Chapel Hill. Encouragement and help from Professor Robert G. Parr are gratefully appreciated, as are discussions with Dr. Shubin Liu.
[1] R. G. Parr and W. Yang, Density-Functional Theory of Atoms and Molecules (Oxford University Press, New York, 1989).

[2] R. M. Dreizler and E. K. U. Gross, Density Functional Theory: An Approach to the Quantum Many-Body Problem (SpringerVerlag, Berlin, 1990).

[3] P. Hohenberg and W. Kohn, Phys. Rev. 136, B864 (1964).

[4] W. Kohn and L. J. Sham, Phys. Rev. 140, A1133 (1965).

[5] J. Harris and R. O. Jones, J. Phys. F 4, 1170 (1974); J. Harris, Phys. Rev. A 29, 1648 (1984).

[6] O. Gunnarsson and B. I. Lundqvist, Phys. Rev. B 13, 4274 (1976).

[7] D. C. Langreth and J. P. Perdew, Phys. Rev. B 21, 5469 (1980).

[8] J. K. Percus, Int. J. Quantum Chem. 13, 89 (1978).

[9] M. Levy, Proc. Natl. Acad. Sci. U.S.A. 76, 6062 (1979).

[10] E. H. Lieb, Int. J. Quantum Chem. 24, 243 (1983).

[11] K. F. Freed and M. Levy, J. Chem. Phys. 77, 396 (1982); J. P. Perdew and M. Levy, Phys. Rev. B 31, 6264 (1985).

[12] M. Levy and J. P. Perdew, Phys. Rev. A 32, 2010 (1985).

[13] S. Liu and R. G. Parr, Phys. Rev. A 53, 2211 (1996).

[14] A. Savin, Phys. Rev. A 52, 1805 (1995).

[15] Y. A. Wang et al., Chem. Phys. Lett. 267, 14 (1997).

[16] R. G. Parr et al., Phys. Rev. A 52, 969 (1995).

[17] E. P. Wigner, Trans. Faraday Soc. 34, 678 (1938).

[18] Y. A. Wang, Chem. Phys. Lett. 268, 76 (1997).

[19] L. C. Wilson and M. Levy, Phys. Rev. B 41, 12930 (1990).

[20] J. A. Alonso and L. A. Girifalco, Solid State Commun. 24, 135 (1977); J. A. Alonso and N. A. Cordero, Int. J. Quantum Chem. Symp. 29, 49 (1995).

[21] O. Gunnarsson et al., Solid State Commun. 24, 765 (1977); O. Gunnarsson and R. O. Jones, Phys. Scr. 21, 294 (1980).
[22] L. H. Thomas, Proc. Cambridge Philos. Soc. 23, 542 (1927); E. Fermi, Z. Phys. 48, 73 (1928).

[23] P. A. M. Dirac, Proc. Cambridge Philos. Soc. 26, 376 (1930).

[24] S. J. Vosko et al., Can. J. Phys. 58, 1200 (1980).

[25] C. F. von Weizsacker, Z. Phys. 96, 431 (1935).

[26] C. H. Hodges, Can. J. Phys. 51, 1428 (1973).

[27] B. Grammaticos and A. Voros, Ann. Phys. (N.Y.) 123, 359 (1979).

[28] D. R. Murphy, Phys. Rev. A 24, 1682 (1981).

[29] L. J. Sham, in Computational Methods in Bond Theory, edited by P. J. Marcus et al. (Plenum, New York, 1971), p. 458.

[30] E. K. U. Gross and R. M. Dreizler, Z. Phys. A 302, 103 (1981).

[31] H. Ou-Yang and M. Levy, Int. J. Quantum Chem. 40, 379 (1991).

[32] J. P. Perdew and Y. Wang, Phys. Rev. B 33, 8800 (1986); 40, 3399(E) (1989); J. P. Perdew, in Electronic Structure of Solid '91, edited by P. Ziesche and H. Eschrig (Akademie-Verlag, Berlin, 1991).

[33] A. D. Becke, J. Chem. Phys. 84, 4524 (1986); 85, 7584 (1986); Phys. Rev. A 38, 3098 (1988).

[34] A. E. DePristo and J. D. Kress, J. Chem. Phys. 86, 1425 (1987).

[35] E. Engel et al., Z. Phys. D 23, 7 (1992).

[36] C. Lee, W. Yang, and R. G. Parr, Phys. Rev. B 37, 785 (1988).

[37] L. C. Wilson, Chem. Phys. 181, 337 (1994).

[38] H. Lee, C. Lee, and R. G. Parr, Phys. Rev. A 44, 44 (1991).

[39] A. J. Thakkar, Phys. Rev. A 46, 4920 (1992).

[40] D. C. Langreth and M. J. Mehl, Phys. Rev. Lett. 47, 446 (1981); Phys. Rev. B 28, 1809 (1983); 29, 2310(E) (1984); C. D. Hu and D. C. Langreth, Phys. Scr. 32, 391 (1985). 